\title{
Results from CLAS and Outlook to CLAS12
}

\author{
M. Ripani \\ INFN Genova, Via Dodecaneso 33, Genova, Italy \\ for the CLAS collaboration
}

\begin{abstract}
In this paper I will illustrate examples of progress in the five main avenues of nucleon structure research in Hall B at JLab: elastic and resonance transition form factors, polarized inelastic scattering, semi-inclusive meson electroproduction, hard exclusive reactions and non-strange baryon spectroscopy. The $12 \mathrm{GeV}$ machine and detector upgrade will offer an unprecedented opportunity to study all these topics in a broader kinematic domain.
\end{abstract}

Keywords: Nucleon Structure, Elastic and Transition Form Factors, Baryon Resonances, Parton Distributions, Inclusive and Semi-inclusive Lepton Scattering, Hard Exclusive Reactions

PACS: $13.40 . \mathrm{Gp}, 13.60 . \mathrm{Fz}, 13.60 . \mathrm{Hb}, 13.60 . \mathrm{Le}, 13.60 . \mathrm{Rj}, 14.20 . \mathrm{Dh}, 14.20 . \mathrm{Gk}, 13.30 . \mathrm{Eg}, 14.40 . \mathrm{Rt}$

\section{INTRODUCTION}

Hall B at JLab, with the CLAS detector, is in the unique position of being able to explore the physics of hadrons from the constituent quark regime up to deep inelastic scattering. Research with CLAS follows several different avenues, and here I will illustrate a few selected topics.

We have now a unified picture of the physics of the nucleon in terms of the information obtained from different reactions. Inclusive electron scattering is interpreted in terms of parton distributions functions (PDFs), representing how the nucleon longitudinal momentum (along the direction of motion) is shared by quarks and gluons. Electromagnetic elastic or $\mathrm{N}-\mathrm{N}^{*}$ transition form factors are connected to the transverse charge distribution in the nucleon. Semi-inclusive meson production involves the transverse momentum of the quarks, therefore their orbital angular momentum, and is parameterized by new objects, the Transverse Momentum Dependent Parton Distribution Functions (TMD PDFs or TMDs). Finally, exclusive hard reactions as Deeply Virtual Compton Scattering (DVCS) and Deeply Virtual Meson Production (DVMP), provide information on yet another family of new objects, the Generalized Parton Distributions (GPD). GPDs provide a 3D picture of the nucleon, in terms of quark/gluon longitudinal momentum distribution and transverse distribution in space. All the above PDFs, regular, TMD and Generalized also carry information about quark polarization. Taken altogether, they provide fundamental information about the composition of the nucleon spin in terms of quark and gluon spin and orbital angular momentum.

With a real photon beam, we don't see partons but bound states of massive objects, the constituent quarks (CQ). However, the spectrum of baryons as calculated in CQ models with three bound quarks and the experimental baryon spectrum do not match: there are more predicted states, which questions the adopted degrees of freedom. A significant part of the CLAS program is to measure several photoproduction channels and their spin 


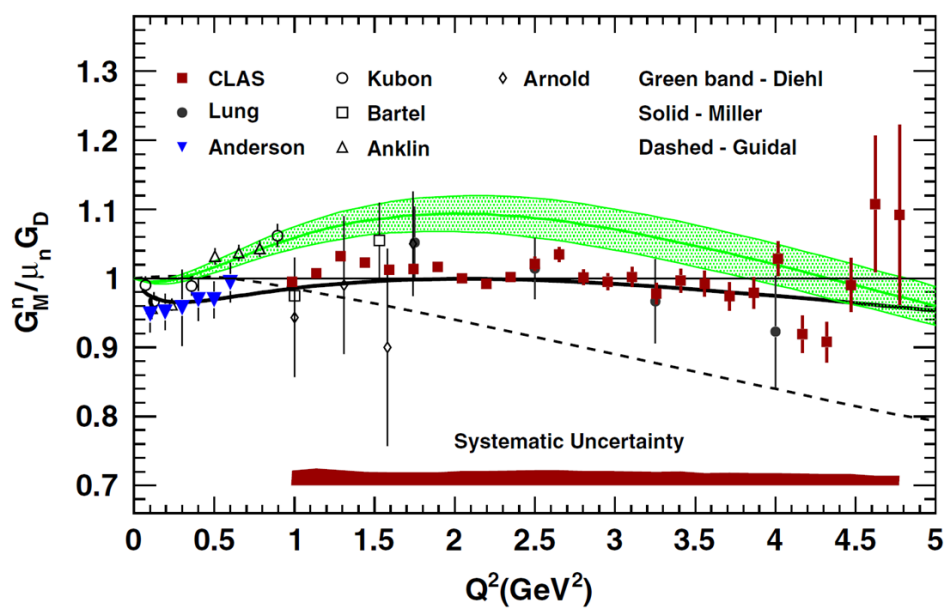

FIGURE 1. Results for $G_{M}^{n} / \mu_{n} G_{D}$ from the CLAS measurement are compared with a selection of previous data and theoretical calculations (see references in [1]). Reprinted figure with permission from [1]. Copyright (2009) by the American Physical Society.

observables. In turn, an intense theoretical effort by the Excited Baryon Analysis Center (EBAC) of JLab, as well as by other theoretical groups worldwide, aims at interpreting the reaction amplitudes in terms of resonance poles. The huge amount of new data, coupled with the ongoing theoretical effort, will allow to clarify the issue of the states predicted and not found to date. Photoproduction is also being used to investigate the meson excited spectrum in multimeson production channels, to look for new states with non- $q \bar{q}$ quantum numbers. I don't have enough space here to report on results on this topic.

\section{ELASTIC AND RESONANCE TRANSITION FORM FACTORS}

Nucleon elastic form factors provide information about the transverse charge distribution inside the nucleon. New generation measurements performed in Hall A at Jefferson Lab already led to a surprise showing that the ratio of electric and magnetic form factors of the proton drops very quickly with momentum transfer. A precise measurement of the magnetic form factor of the neutron $G_{M}^{n}$ [1] was performed with CLAS. As is seen in Fig. $1 G_{M}^{n}$ seems to follow closely the dipole form.

Pion electroproduction with CLAS was used to extract the magnetic transition form factor $G_{M}$ and the multipole ratios $R_{E M}=\frac{\operatorname{Im}\left(E_{1+}\right)}{\operatorname{Im}\left(M_{1+}\right)}, R_{S M}=\frac{\operatorname{Im}\left(S_{1+}\right)}{\operatorname{Im}\left(M_{1+}\right)}$ for the transition $\gamma^{*} \mathrm{p}$ $\rightarrow \Delta(1232) P_{33}$ up to about $6 \mathrm{GeV}^{2}$ momentum transfer $[2,3]$, where $M_{1+}, E_{1+}$ and $S_{1+}$ are notations for the magnetic dipole excitation, the transverse electric quadrupole and the longitudinal electric (Coulomb) quadrupole. These quantities are reported in Fig. 2. It is found that $G_{M}$ continues to fall faster than the dipole. The ratio $R_{E M}$ is different from zero, this being a direct indication of residual interactions breaking the $S U(6) \times O(3)$ spin-flavor-space symmetry. At high momentum transfer, according to Ref. [5] $R_{E M}$ should approach unity, while $R_{S M}$ should approach a constant. Neither of these two 

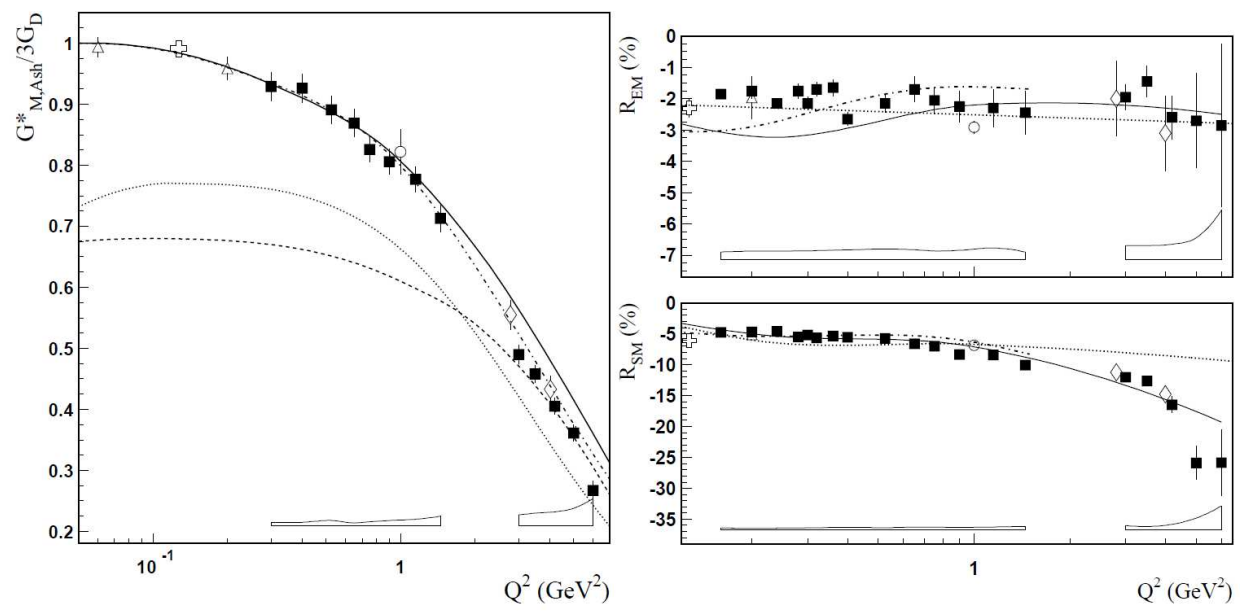

FIGURE 2. Left panel: $G_{M}$ for the $\Delta(1232) P_{33}$, relative to $3 \mathrm{G}_{D}$ [3]. Right panel: the ratios $R_{E M}, R_{S M}$. The full boxes are the CLAS data [3]. The bands show the model uncertainties. Also shown are the results from MAMI - open triangles, MIT/BATES - open crosses, JLab/Hall C - open rhombuses, and JLab/Hall A - open circles. Curves represent various theoretical calculations and fits (see references in [2, 3, 4]). Reprinted figure with permission from [4]. Copyright (2009) by the American Physical Society.

predictions is supported by the data, indicating that apparently these QCD asymptotia are still farther away.

The $\mathrm{N}^{*}$ program with CLAS provided also an impressive body of new data about $\pi^{+}$electroproduction [6]. The $\pi^{+}$electroproduction data, both for cross sections and for beam spin asymmetries, were analysed together with the CLAS $\pi^{0}$ [2] and double pion production data [7], to extract information on the $P_{11}(1440)$ (Roper) resonance excitation. In the past, speculations have been made that this state may be a hybrid baryon, built out of three CQ plus a gluon. In this picture, the longitudinal coupling $S_{1 / 2}$ should be exactly zero [8]. The analysis in [2] was extended to higher $\mathrm{Q}^{2}$ by using the most recent CLAS data [4]. Both in [2] and [4] the analysis was performed by using the UIM approach as well as a Dispersion Relation (DR) model. The two analyses gave very close results and were averaged to present the final points. Fig. 3 shows the extracted values of $A_{1 / 2}$ and $S_{1 / 2}$, together with theoretical calculations. The hybrid picture is ruled out definitevely by the CLAS data indicating a non-zero $S_{1 / 2}$. Non-relativistic CQ Models were known to not reproduce the sign of $A_{1 / 2}$ at $\mathrm{Q}^{2}=0$ and to not show any zero-crossing [2]. Relativistic CQ Models [9, 10] provide both the correct sign and the zero-crossing.

\section{POLARIZED INCLUSIVE SCATTERING}

The spin structure of the nucleon has been investigated for more than three decades using lepton and photon beams. Measurements of the spin-dependent structure functions $g_{1}$ and $g_{2}$ have been performed at large $Q^{2}$ (Deep Inelastic Scattering or DIS region) at SLAC, CERN, and DESY, providing information on the spin and momentum of the 

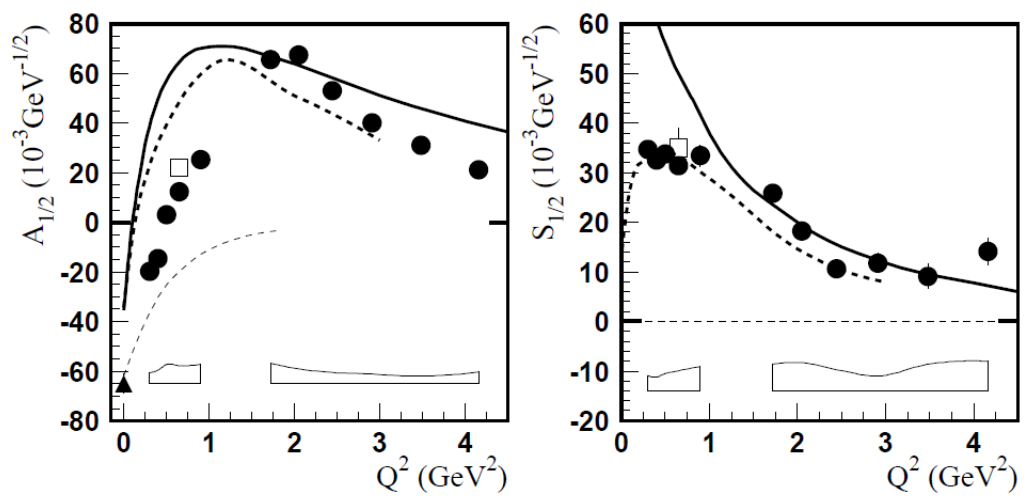

FIGURE 3. Helicity amplitudes for the $\gamma^{*} \mathrm{p} \rightarrow N(1440) P_{11}$ transition. The full circles are the CLAS data $[6,2,7,4]$. The bands show the model uncertainties. The open boxes are the results of the combined analysis of CLAS single $\pi$ and $2 \pi$ electroproduction data [2]. The full triangle at $Q^{2}=0$ is the RPP [11] estimate. Curves represent various theoretical calculations (see references in $[6,2,7,4]$. Reprinted figure with permission from [4]. Copyright (2009) by the American Physical Society.

partons at very high momentum transfer. On the contrary, much less was known in the low momentum transfer region $\left(Q^{2}<1-2 \mathrm{GeV}^{2}\right)$, where lattice QCD should be applied instead of perturbative QCD. A broad program to measure the inclusive spin structure of the nucleon via polarized electron scattering off a polarized target was conducted between 1998 and 2006 with CLAS [12]. Fig. 4, shows the impressive effect of the new CLAS measurements on our knowledge of the polarized structure function $g_{1}$. The low $Q^{2}$ CLAS data had a significant impact on the polarized gluon density extracted in next-to-leading order fits [13]. At $\mathrm{x}>0.2$ a factor 2 to 4 reduction in the polarized gluon density uncertainty is achieved, while an additional improvement at small $x$ is obtained when the new COMPASS data on deuterium are used in the fit. Overall the extracted polarized gluon density is much smaller than what is required to explain the shortfall from the polarized quark densities.

\section{SEMI-INCLUSIVE MESON ELECTROPRODUCTION}

Semi-inclusive deep inelastic scattering (SIDIS), studied at CLAS by detecting a meson in coincidence, combined with the use of polarization, provides an essential new tool in understanding parton dynamics. In the QCD factorization picture the process is described by the product of: new, Transverse Momentum dependent, PDFs (TMDs) $[14,15]$, that are sensitive to the orbital motion of quarks and to the correlations of spin and transverse momentum of quarks in the nucleon; quark fragmentation functions. TMDs give rise to new observables revealed in azimuthal distributions of the final state meson. Many experiments worldwide are currently trying to pin down various TMD effects through semi-inclusive deep-inelastic scattering (in experiments such as HERMES at DESY, COMPASS at CERN, CLAS and Hall-A at Jefferson Lab) polarized proton-proton collisions (PHENIX, STAR and BRAHMS at RHIC), and electronpositron annihilation (Belle at KEK). In Fig. 5, the SIDIS structure function $\mathrm{H}_{2}$, anal- 


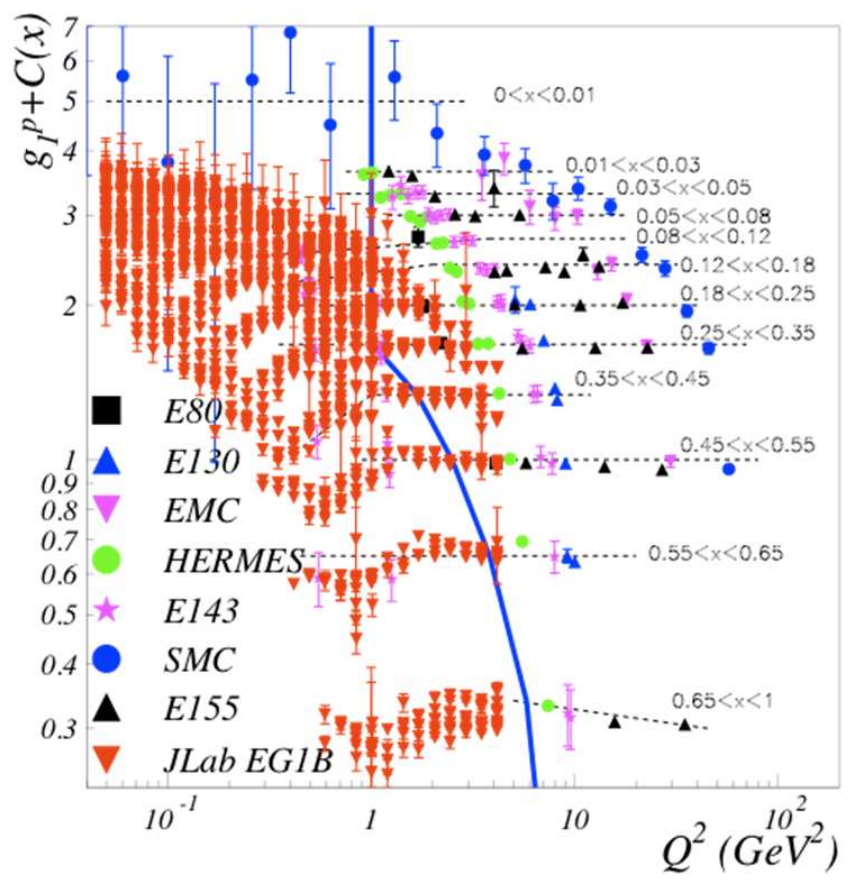

FIGURE 4. The CLAS data [12] (inverted triangles) for the structure function $g_{1}$, as a function of the momentum transfer $\mathrm{Q}^{2}$, for several bins in Bjorken $x$, in comparison to the world data, including other JLab experiments

ogous of $F_{2}$ in DIS, as measured in CLAS, is reported and compared to LO and NLO pQCD calculations [16]. The agreement is satisfactory, showing that also in the kinematics of CLAS at $6 \mathrm{GeV}$ beam energy, the deep inelastic description seems to be working fine. A broader program for the measurement of several polari zation observables, both with pions and kaons, has been proposed for the current $6 \mathrm{GeV}$ kinematics and for the future upgrade of the machine to $12 \mathrm{GeV}$.

\section{DEEPLY VIRTUAL COMPTON SCATTERING AND GPDS}

In Deeply Virtual Compton scattering (DVCS) $\left(\gamma^{*} p \rightarrow \gamma p\right)$, a quark is picked up from the nucleon by the virtual photon, then goes back to the nucleon with a different momentum after emitting a real photon. DIS can be represented by a similar process, but with the quark returning to the nucleon with the same momentum it had before. It can be shown that, owed to this difference, a new generation of PDFs, the Generalized Parton Distributions (GPDs) $H, \tilde{H}, E, \tilde{E}(x, \xi, t)$ has to be introduced to parameterize DVCS [17]. GPDs depend on three kinematic variables instead of one (besides $Q^{2}$ ) and generally must be integrated over the longitudinal momentum fraction of the quark $x$. $t$, the squared 4-momentum transfer to the target, and $\xi=f\left(x_{B}\right)$ are fixed by the kinematics. GPDs can be translated into a 3D imaging of the nucleon in transverse coordinate space and 


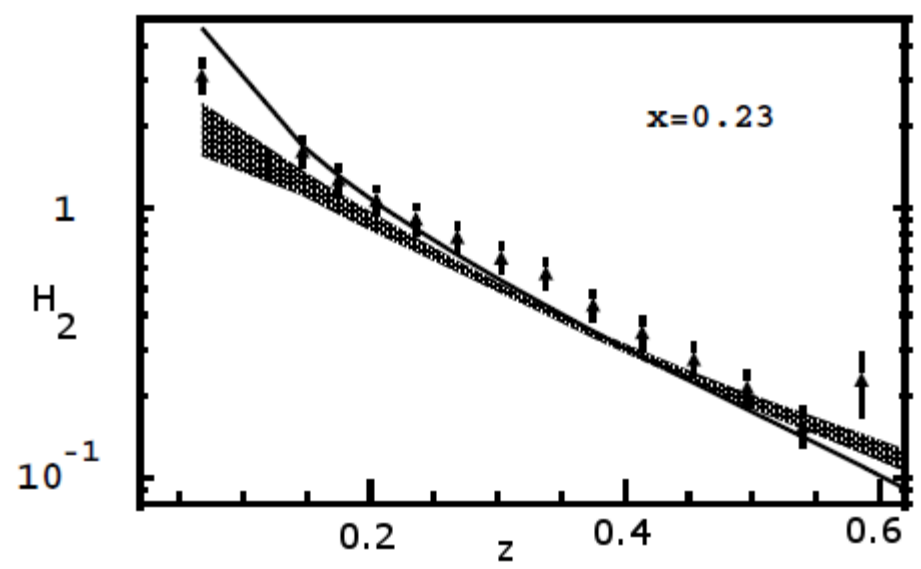

FIGURE 5. The $\mathrm{z}$-dependence of $H_{2}$ at $\mathrm{Q}^{2}=2.37(\mathrm{GeV} / \mathrm{c})^{2}$. The CLAS data [16] are shown by full triangles. The error bars give statistical and systematic uncertainties combined in quadrature. Solid line: LO pQCD calculation; shaded area: NLO calculation with its uncertainties (See [16] and references therein). Reprinted part of figure with permission from [16]. Copyright (2009) by the American Physical Society.

longitudinal momentum space at once, at variance with form factors, DIS and SIDIS where only one or two dimensions are accessed.

An unprecedented set of Deeply Virtual Compton Scattering data was accumulated as part of the CLAS physics program, which allowed the first systematic and precise exploration of a sensitive observable, the beam-spin asymmetry of the reaction $\vec{e} p \rightarrow$ ep $\gamma$ [18]. This asymmetry arises mainly from the interference between the Bethe-Heitler (BH) and DVCS processes (where the photon is emitted by the electron or by the target nucleon, respectively). At leading twist, it is primarily sensitive to the imaginary part of the DVCS amplitude and thus to a specific linear combination of the proton GPDs $H$, $\tilde{H}$ and $E$. Model calculations have been used to extract the GPDs in global fits to the data. New upcoming data including other polarization observables, as well as data on the neutron, will help constrain the fits and extract the full set of GPDs.

\section{THE SEARCH FOR MISSING BARYONS}

SU(6) symmetric quark models predict more states than have been found in experiments. This discrepancy may be explained either by QCD mixing effects moving coupling strength from the $\pi N$ channel to multipion or hyperon channels; or by a reduced set of degrees of freedom, as in the quark-diquark picture, resulting in a fewer number of states. Search for the states still missing in the experimental observation is therefore crucial in understanding the basic degrees of freedom in baryon structure. Moreover, many high-lying and poorly known baryon states decay predominantly in multipion or hyperon production channels [11]. Therefore, the measurement of photoproduction cross sections and polarization observables of several exclusive channels can provide both a better knowledge of the heavier states and a roadmap for discovering new states. Such a 


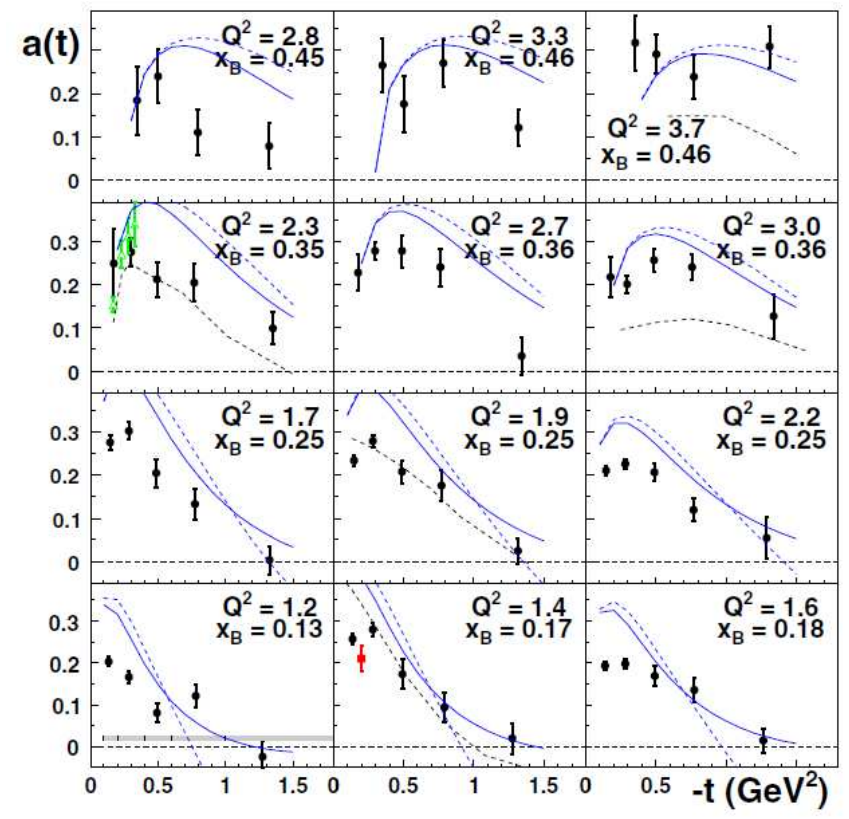

FIGURE 6. Spin asymmetry for the reaction $\gamma^{*} p \rightarrow \gamma p$ at $90^{\circ}$ as a function of $\mathrm{t}$. Each individual plot corresponds to a bin in $\left(\mathrm{x}_{B}, \mathrm{Q}^{2}\right)$. Systematic uncertainties and bin limits are illustrated by the gray band in the lower left plot. Circles and squares: recent [18] and previous data from CLAS. Triangles: Hall A data at similar, but not equal, values of $\left\langle x_{B}\right\rangle$ and $\mathrm{Q}^{2}$. The curves correspond to various theoretical calculations (see references in [18]). Reprinted part of figure with permission from [18]. Copyright (2008) by the American Physical Society.

program is being conducted in Hall B by using linearly or circularly polarized photons and polarized targets. Fig. 7 shows preliminary data about the polarization observable $E$ in $\pi^{+}$photoproduction off the proton. The $E$ asymmetry results from flipping the helicity of photons hitting protons polarized along the beam direction. The angular dependence of this observable changes significantly as a function of the hadronic mass $W$, a fundamental feature in constraining model fits of resonance production.

\section{CONCLUSIONS}

The powerful combination of the continuous, polarized, $6 \mathrm{GeV}$ electron beam at JLab and the large acceptance CLAS detectors allowed to pursue several different physics topics, ranging from baryon and meson spectroscopy to nucleon form factors, to hard semi-inclusive and exclusive reactions. This is offering a unique opportunity to study QCD from the truly non-perturbative discrete states, to the microscopic partonic structure. The upcoming upgrade of the machine to $12 \mathrm{GeV}$, together with the upgrade of the CLAS detector to CLAS12, will provide the unique combination of wide kinematic coverage, high beam intensity (luminosity), high energy, high polarization, and advanced detection capabilities necessary to extend the study of spectroscopy and form factors to higher masses and momentum transfers, and to extend the study of the new parton distributions to a broader kinematic regime. 

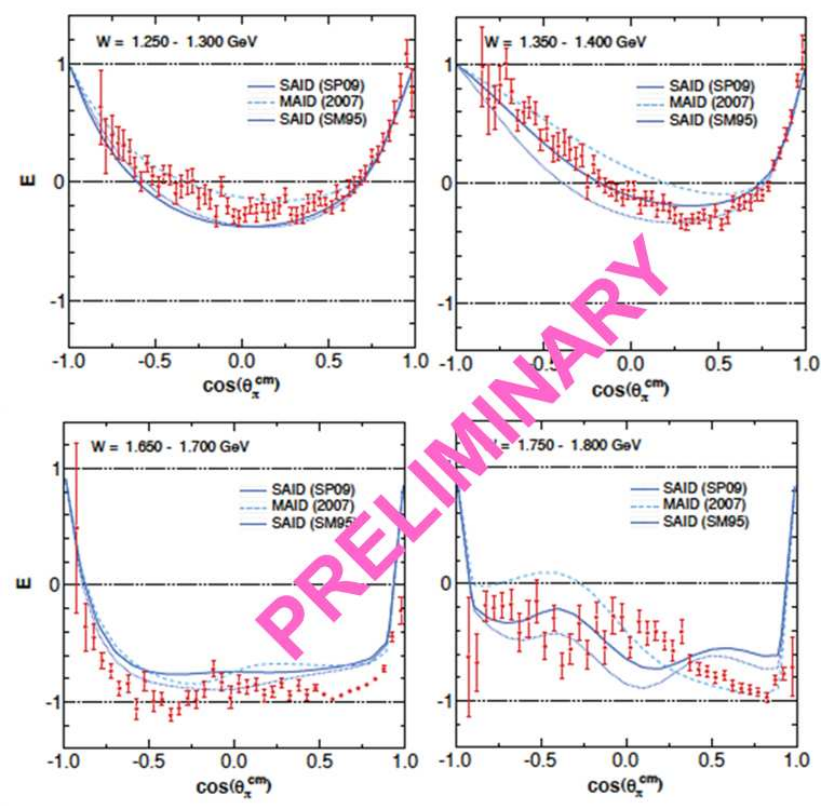

FIGURE 7. Spin observable $E$ for $\pi^{+}$photoproduction. The points are preliminary data from CLAS, as a function of the pion CM angle and for four different hadronic mass $W$ intervals. Curves are various partial wave fits as reported in the legend.

\section{REFERENCES}

1. J. Lachniet et al., Phys.Rev. Lett. 102, 192001 (2009).

2. I. Aznauryan et al., Phys. Rev. C71, 015201 (2005).

3. K. Joo et al., Phys.Rev. Lett. 88, 122001 (2002); K. Joo et al., Phys. Rev. C68, 032201(R) (2003); M. Ungaro et al., Phys.Rev. Lett. 97, 112003 (2006).

4. I G. Aznauryan et al. Phys. Rev. C80, 055203 (2009).

5. S. J. Brodsky, G. F. de Teramond, Phys. Lett. B582, 211 (2004).

6. K. Joo et al., Phys. Rev. C70, 042201 (2004); K. Joo et al., Phys. Rev. C72, 058202 (2005); H. Egiyan et al., Phys. Rev. C73, 025204 (2006); K. Park et al., Phys. Rev. C77, 015208 (2008).

7. I. G. Aznauryan et al., Phys. Rev. C72, 045201 (2005).

8. Z.-p. Li et al., Phys. Rev. D46, 70 (1992).

9. S. Capstick and B. D. Keister, Phys. Rev. D51, 3598 (1995).

10. I. G. Aznauryan, Phys. Rev. C76, 025212 (2007).

11. C. Amsler et al., "Review of Particle Properties", Phys. Lett. B667, 2008.

12. R. Fatemi et al., Phys. Rev. Lett. 91, 222002 (2003); V. Darmawardane et al., Phys. Lett. B641, 11 (2006); Y. Prok et al., Phys. Lett. B672, 12 (2009).

13. E. Leader, A. Sidorov, and D. Stamenov, Phys. Rev. D75, 074027 (2007).

14. P J. Mulders and R. D. Tangerman, Nucl. Phys. B461, 197 (1996).

15. A. Bacchetta et al., JHEP 0702, 093 (2007).

16. M. Osipenko et al., Phys. Rev. D80, 032004 (2009).

17. D. Müller et al., Fortschr. Phys. 42, 101 (1994); K. Goeke, M. V. Polyakov, and M. Vanderhaeghen, Prog. Part. Nucl. Phys. 47, 401 (2001).

18. F. X. Girod et al., Phys. Rev. Lett. 100, 162002 (2008). 